PROCEEDINGS OF THE

AMERICAN MATHEMATICAL SOCIETY

Volume 127, Number 3, March 1999, Pages 907-913

S 0002-9939(99)04568-2

\title{
LINDELÖF PROPERTY AND ABSOLUTE EMBEDDINGS
}

\author{
A. BELLA AND I. V. YASCHENKO \\ (Communicated by Alan Dow)
}

\begin{abstract}
It is proved that a Tychonoff space is Lindelöf if and only if whenever a Tychonoff space $Y$ contains two disjoint closed copies $X_{1}$ and $X_{2}$ of $X$, then these copies can be separated in $Y$ by open sets. We also show that a Tychonoff space $X$ is weakly $C$-embedded (relatively normal) in every larger Tychonoff space if and only if $X$ is either almost compact or Lindelöf (normal almost compact or Lindelöf).
\end{abstract}

\section{INTRODUCTION AND RESULTS}

Notations and terminology follow [En]. Unless otherwise stated, all spaces are assumed to be Tychonoff (and $T_{1}$ ). By $\beta X$ we denote the Stone-Cech extension of the space $X$. Recall that the Lindelöf number of a space $X$, denoted by $l(X)$, is the smallest cardinal $\tau$ such that every open cover of $X$ has a subcover of size not greater than $\tau$. If $l(X) \leq \omega$, then $X$ is called Lindelöf. For a non-compact space $X$ put $\lambda(X)=\min \left\{|A|: A \subset X\right.$ and $\mathrm{Cl}_{X} A$ is not compact $\} . \lambda(X)$ might be called the index of boundedness of $X$, since $\lambda(X) \geq \omega_{1}$ if and only if $X$ is $\omega$-bounded.

Observe that if $\mu<\lambda(X)$, then each subset $A \subset X$ of cardinality $|A| \leq \mu$ has a complete accumulation point, so $X$ is initially $\mu$-compact (i.e., every open cover of $X$ of size not bigger than $\mu$ has a finite subcover) [St, Theorem 2.2].

Two subsets $A$ and $B$ of a space $X$ are said to be completely separated if there is a continuous function $f: X \rightarrow \mathbb{R}$ so that $f(A)=\{0\}$ and $f(B)=\{1\}$.

The main result of the paper is the following theorem, proving true the conjecture of Arhangel'skij [AT, Problem 2].

Theorem 1.1. For a Tychonoff space $X$ the following conditions are equivalent:

1. $X$ is Lindelöf.

2. If a Tychonoff space $Y$ contains two disjoint closed copies $X_{1}$ and $X_{2}$ of $X$, then these copies can be separated in $Y$ by open sets.

Hewitt was the first to consider the problem of whether a space $X$ is $C$-embedded into every larger Tychonoff space $Y$ (that is, every continuous function on $X$ can be extended to a continuous function on $Y$ ). It was proved by Hewitt and Smirnov $[\mathrm{He}],[\mathrm{Sm}]$ (see also [GJ, Ex. 6J]) that this is the case if and only if $X$ is almost

Received by the editors November 14, 1996 and, in revised form, June 26, 1997.

1991 Mathematics Subject Classification. Primary 54A35, 54D20.

Key words and phrases. Lindelöf space, normal space, relative topological property, embedding, almost compact space.

This work was done while the second author was visiting Catania University. He is grateful to Italian colleagues for generous hospitality and to CNR for financial support.

(C)1999 American Mathematical Society 
compact, i.e. $|\beta X \backslash X| \leq 1$, or, equivalently, for every two disjoint completely separated closed subsets of $X$ at least one is compact.

Later, R.Blair proved that a space $X$ is absolutely $\nu$-embedded (that is, $\nu X \subset \nu Y$ whenever $X \subset Y$ ) if and only if $X$ is either realcompact or almost compact [Bl]. R.Blair, A.Hager and D.Johnson proved that a space $X$ is absolutely $z$-embedded (that is, each zero-set of $X$ can be extended to a zero-set of $Y$ whenever $X \subset Y$ ) if and only if $X$ is either Lindelöf or almost compact. They also provided several interesting characterizations of such spaces $[\mathrm{Bl}],[\mathrm{BH}],[\mathrm{HJ}]$. E.g. these are the spaces so that the $C(X)$ - ring of continuous functions on $X$ - has no proper subalgebra which contains constant functions, separates points and closed sets, is closed under uniform convergence and is closed under inversion in $C(X)$ [HJ, Theorem 3]. Another result, which will be usefull for us, says that if every pair of disjoint non-compact zero-sets of $X$ consists of Lindelöf sets, then $X$ itself is either Lindelöf or almost compact (and hence absolutely $z$-embedded) [BH, Theorem 4.1].

Recently, the following weaker version of $C$-embedding has attracted attention, in particular in the theory of relative topological properties.

Definition 1.2 (Arhangel'skij). A subspace $X$ is weakly $C$-embedded into a space $Y$ if every continuous real-valued function on the space $X$ can be extended to a real-valued function on $Y$, which is continuous in all points of $X$.

Notice that, while $C$-embedding and $C^{*}$-embedding (if we assume that only bounded functions must have an extension) are, in general, different notions, weak $C$-embedding coincides with weak $C^{*}$-embedding. (Just consider an unbounded function as a function $f: X \rightarrow(-1,1)$, extend it to the function $\tilde{f}: Y \rightarrow[-1,1]$, which is continuous in all points of $X$ and note that $\mathrm{Cl}_{Y} f^{-1}\{-1,1\} \cap X=\emptyset$, so we can modify $\tilde{f}$ to have value 0 in all points $y$ with $f(y)= \pm 1$ without any loss of continuity at the points of $X$.)

Every dense embedding into a Tychonoff space is a weak $C$-embedding [Ar], but in the case of non-dense embedding this concept is far from being trivial.

Arhangel'skij [Ar] observed that if $X$ is almost compact or Lindelöf, then every embedding of $X$ into a larger Tychonoff space is a weak $C$-embedding. Surprisingly, these are the only possibilities. Precisely, the following theorem, answering the question of Arhangel'skij [Ar, problem 15], holds.

Theorem 1.3. A space $X$ is weakly $C$-embedded into every larger Tychonoff space $Y$, containing $X$ as a closed subspace, if and only if $X$ is either almost compact, or Lindelöf.

In [Ar] it was also asked to characterize the spaces which are (strongly) relatively normal in every larger Tychonoff space (Problems 49 and 50); see also [AT, Problems 3 and 4]. A subspace $X \subset Y$ is called (strongly) relatively normal in $Y$ if every two disjoint closed in $Y$ (closed in $X$ ) subsets of $X$ can be separated by open in $Y$ subsets (see [Ar] for a recent survey on relative topological properties).

In $[\mathrm{AT}]$ it was observed that all normal almost compact spaces and in $[\mathrm{AG}]$ that all Lindelöf spaces are strongly relatively normal in every larger Tychonoff space.

We prove here that "the converse" is true.

Theorem 1.4. If $X$ is a Tychonoff space, then the following conditions are equivalent:

1. $X$ is either normal and almost compact or Lindelöf. 
2. $X$ is strongly normal in every Tychonoff space.

3. $X$ is normal in every Tychonoff space.

4. $X$ is normal in every Tychonoff space, containing $X$ as a closed subspace.

5. $X$ is normal and every pair of non-compact disjoint closed subsets of $X$ consists of Lindelöf sets.

\section{Proofs}

Lemma 2.1. Let $P$ and $Q$ be disjoint closed sets of a space $X$. If any Tychonoff space $Y$, which contains $X$ as a closed subspace, has two disjoint open sets separating $P$ and $Q$, then either both $P$ and $Q$ are Lindelöf, or one of them is compact.

Proof. Take two disjoint closed subsets $P$ and $Q$ of $X$ and suppose that both are non-compact and $P$ is not Lindelöf.

Lemma 2.2. $\lambda(Q) \geq l(P)>\omega$.

Proof of Lemma 2.2. Assuming the contrary, there is $A \subset Q$ so that $\mathrm{Cl}_{Q} A$ is noncompact and $|A|<l(P)=\tau$.

Since $|A|<\tau$, there is an open cover $\gamma$ of the space $P$ having no subcover of size $\leq|A|$. We may assume that $\gamma$ has no subcover of size less than $|\gamma|=\tau^{\prime}$. Consider $\alpha P=\mathrm{Cl}_{\beta X} P$ - a compactification of $P$. Then it is easy to see that there is a $Z \subset \alpha P$ such that $Z$ can be separated from $P$ in $\alpha P$ by a $G_{\tau^{\prime}}$-set, but cannot be separated from $P$ by any $G_{\beta}$-set for $\beta<\tau^{\prime}$ [En, Ex. 3.12.24]. Since $P$ is closed in $X, \alpha P \cap X=P$ and $Z \subset(\beta X \backslash X)$. Since $\mathrm{Cl}_{Q} A$ is not compact and $Q$ is closed in $X$, there is a point $a \in \beta X \backslash X$ so that $a \in \mathrm{Cl}_{\beta X} A$. Consider a compactification $C$ of the space $X$, obtained from $\beta X$ by identifying the compact set $Z \cup\{a\}$ to a point $b$.

Let $S=P \cup Q \cup\{b\} \subset C$ and $T=S \times S \backslash\{(b, b)\}$. We claim that the closed subsets of $T, P \times\{b\}$ and $\{b\} \times Q$ cannot be separated in $T$ by open sets. Indeed, let $U$ be an open subset of $T$ such that $\{b\} \times Q \subset U$. For every $x \in A \subset Q$ there exist open neighborhoods $V_{x}$ and $W_{x}$ of the points $b$ and $x$ in $S$ such that $V_{x} \times W_{x} \subset U$. The set $G=\bigcap\left\{V_{x}: x \in A\right\}$ is a $G_{\beta}$ set containing $b$ with $\beta=|A|<\tau^{\prime}$. Thus $G$ meets $P$, say for instance in a point $y$. It follows that $\{y\} \times A \subset U$ and, since in $S$ the point $b$ is in the closure of $A$, we actually have $(y, b) \in \mathrm{Cl}_{T}\{y\} \times A \subset \mathrm{Cl}_{T} U$. Hence we cannot have $P \times\{b\} \cap \mathrm{Cl}_{T} U=\emptyset$.

As a consequence, we see that $X$ is not normal in the quotient space of $X \oplus T$, obtained by identifying $P$ with $P \times\{b\}$ and $Q$ with $\{b\} \times Q$. This contradiction finishes the proof of the Lemma 2.2 .

Lemma 2.3. $l(Q) \geq \lambda(Q)$, in particular $Q$ is not Lindelöf.

Proof of Lemma 2.3. If $l(Q)=\tau<\lambda(Q)$, then the closure in $Q$ of any set of cardinality at most $\tau$ is compact. In particular, $Q$ is initially $\tau$-compact and hence compact since $l(Q)=\tau$. But this contradicts the assumption that $Q$ is not compact.

Lemma 2.4. $\lambda(P) \geq l(Q)$.

Proof of Lemma 2.4. Use the same argument as in the proof of Lemma 2.2.

Lemma 2.5. $\lambda(P)=l(P)=\lambda(Q)=l(Q)=\tau>\omega$ and $\tau$ is a regular cardinal. 
Proof of Lemma 2.5. $\lambda(P)=l(P)=\lambda(Q)=l(Q)=\tau>\omega$ follows from Lemmas 2.2-2.4. Suppose $c f(\tau)=\mu<\tau$, i.e. $\tau=\sup \left\{a_{\alpha}: a<\mu\right\}$. Since $P$ is not Lindelöf, we can take an uncountable open cover $\gamma$ of the space $P$, having no subcover of size less than $|\gamma|$. We claim that $|\gamma|=\tau$. Since $l(P)=\tau,|\gamma| \leq \tau$. If $|\gamma|<\tau$, then, as $P$ is $|\gamma|$-initially compact, $\gamma$ would have a finite subcover. So, $|\gamma|=\tau$. Well order $\gamma$ as $\gamma=\left\{U_{\alpha}: \alpha<\tau\right\}$. For every $\alpha<\mu$ let $W_{\alpha}=\bigcup\left\{U_{\beta}: \beta<a_{\alpha}\right\}$. Now, $\left\{W_{\alpha}: \alpha<\mu\right\}$ is a cover of $P$ of size less than $\tau$, so it has a finite subcover. Hence $\gamma$ has a subcover of size less than $\tau$-a contradiction.

Now we continue the proof of Lemma 2.1.

Preliminary construction. By transfinite induction we shall construct in $P$ something like an enlarged closed copy of the ordinal $\tau$.

Since $l(P)=\lambda(P)=\tau$, the proof of Lemma 2.5 shows that there is an open cover $\gamma$ of size $\tau$ having no subcover of size less than $\tau$. Hence by the same argument as in the proof of Lemma 2.2, there is a compact $Z \subset \alpha P=\mathrm{Cl}_{\beta X} P$ such that $Z$ can be separated from $P$ in $\alpha P$ by a $G_{\tau}$-set, but cannot be separated from $P$ by any $G_{\beta}$-set for $\beta<\tau$.

By transfinite induction define for every $\alpha<\tau$ a compact subset $F_{\alpha} \subset P$ and an open in $\alpha P$ neighborhood $W_{\alpha} \supset Z$ in such a way that

(1) $F_{\alpha} \subset \bigcap\left\{\mathrm{Cl}_{\alpha P} W_{\beta}: \beta<\alpha\right\}$,

(2) $\mathrm{Cl}_{\alpha P} W_{\alpha} \cap \bigcup\left\{F_{\beta}: \beta \leq \alpha\right\}=\emptyset$, in particular all $F_{\alpha}$ are disjoint,

(3) if $\alpha$ is a non-limit ordinal, then $F_{\alpha}$ is a one-point-set $F_{\alpha}=\left\{x_{\alpha}\right\}$,

(4) if $\alpha$ is a limit ordinal, then

$$
F_{\alpha}=\mathrm{Cl}_{P} \bigcup\left\{F_{\beta}: \beta<\alpha\right\} \backslash \bigcup\left\{F_{\beta}: \beta<\alpha\right\},
$$

(5) $d\left(\bigcup\left\{F_{\beta}: \beta<\alpha\right\}\right) \leq \alpha$,

(6) for every $\alpha<\tau, \bigcup\left\{F_{\beta}: \beta \leq \alpha\right\}$ is a compact subset of $P$.

Take an arbitrary $x \in P$ as $x_{0}$, and let $F_{0}=\left\{x_{0}\right\}$ and $W_{0}$ be an arbitrary neighborhood of $Z$ so that $x_{0} \notin \mathrm{Cl}_{\alpha P} W_{0}$.

Let $\alpha<\tau$ and suppose that for all $\beta<\alpha$ we have $F_{\beta}$ and $W_{\beta}$ satisfying conditions (1)-(6). Consider two possibilities:

I. $\alpha=\alpha^{\prime}+1$. Since $Z$ cannot be separated from $P$ by $G_{\alpha^{\prime}}$-sets, there is a point $x_{\alpha} \in P \cap \bigcap\left\{W_{\beta}: \beta \leq \alpha^{\prime}\right\}$. Let $F_{\alpha}=\left\{x_{\alpha}\right\}$. Clearly conditions (1),(3),(5),(6) are satisfied for $\alpha$. From (6) it follows that there is an open neighborhood $W_{\alpha}$ of $Z$ so that $\mathrm{Cl}_{\alpha P} W_{\alpha} \cap \bigcup\left\{F_{\beta}: \beta \leq \alpha\right\}=\emptyset$. Of course, even (2) is satisfied and we are done for $\alpha$.

II. $\alpha$ is limit ordinal. Let $F_{\alpha}^{\prime}=\mathrm{Cl}_{P} \bigcup\left\{F_{\beta}: \beta<\alpha\right\}$. Since (5) is true for each $\beta<\alpha$ and $\alpha<\lambda(P)$, it follows that $F_{\alpha}^{\prime}$ is a compact subset of $P$. From (1), (2) and (6) for each $\beta<\alpha$ we have

$$
F_{\alpha}=F_{\alpha}^{\prime} \backslash \bigcup\left\{F_{\beta}: \beta<\alpha\right\}=F_{\alpha}^{\prime} \cap \bigcap\left\{\mathrm{Cl}_{\alpha P} W_{\beta}: \beta<\alpha\right\} .
$$

In particular, $F_{\alpha}$ is compact. Since $F_{\alpha}^{\prime}$ is a compact subset of $P$, there is an open neighborhood $W_{\alpha}$ of $Z$ so that $\mathrm{Cl}_{\alpha P} W_{\alpha} \cap F_{\alpha}^{\prime}=\emptyset$. Now all conditions (1)-(6) are clearly satisfied and we are done for $\alpha$.

Clearly, the $W_{\alpha}$ 's may be chosen in such a way that in addition $Z=\bigcap\left\{\mathrm{Cl}_{\alpha P} W_{\alpha}\right.$ : $\alpha<\tau\}$. It then follows that $F=\bigcup\left\{F_{\alpha}: \alpha<\tau\right\}$ is closed in $P$. 
The preliminary construction is now complete. We can think about $F$ as a closed copy of $\tau$ in $P$ where all non-limit ordinals are usual points, but all limit ordinals are enlarged to (maybe non-trivial) compact sets.

Next, apply the same method to get a similar "copy of $\tau$ " $G=\bigcup\left\{G_{\alpha}: \alpha<\tau\right\}$ in $Q$.

Now we are ready to embed $X$ in a space $Y$ in such a way that the sets $P$ and $Q$ cannot be separated by open sets in $Y$. Let $T=(\tau+1) \times(\tau+1) \backslash\{(\tau, \tau)\}$. We shall use the well known fact that closed copies of $\tau$ in $T$ such as the diagonal $\Delta \subset T$ and $\tau \times\{\tau\}$ cannot be separated by open sets in $T$ (see [Wa, Lemma 2.1.12]).

The idea of our construction is to "replace" here $\Delta$ and $\tau \times\{\tau\}$ by $F$ and $G$ and then "glue" the resulting space to $X$.

Let $T^{\prime}$ be the set of all points in $\tau \times \tau \backslash \Delta$ having both coordinates non-limit. The space $Y$ we are looking for is $X \cup T^{\prime}$ equipped with the following topology:

(1) All points of $T^{\prime}$ are isolated.

(2) All points $x \in X \backslash(F \cup G)$ have usual neighborhood base in $X$.

(3) All points $x \in \bigcup\left\{F_{\alpha}: \alpha\right.$ is non-limit $\}$ have usual neighborhood base in $X$.

(4) Let $x \in F_{\alpha}$ and $\alpha$ be a limit ordinal. For every open in $X$ a neighborhood $W$ of $x$ define a neighborhood $W^{\prime}$ of $x$ in $Y$ as

$$
W^{\prime}=W \cup\left\{(a, b) \in T^{\prime}: F_{a} \subset W \text { and } F_{b} \subset W\right\} .
$$

Notice that, in the above formula, $a$ and $b$ are non-limit ordinals, so $F_{a}$ and $F_{b}$ are one-point-sets.

(5) Let $x \in G_{\alpha}$ and $\alpha$ be a non-limit ordinal. For every open in $X$ neighborhood $W$ of $x$ and every $b<\tau$ define a neighborhood $W_{b}^{\prime}$ of $x$ in $Y$ as

$$
W_{b}^{\prime}=W \cup\left\{(\alpha, c) \in T^{\prime}: c>b\right\} .
$$

(6) Let $x \in G_{\alpha}$ and $\alpha$ be a limit ordinal. For every open in $X$ neighborhood $W$ of $x$ and every $b<\tau$ define a neighborhood $W_{b}^{\prime}$ of $x$ in $Y$ as

$$
W_{b}^{\prime}=W \cup\left\{(a, c) \in T^{\prime}: G_{a} \subset W \text { and } c>b\right\} .
$$

Notice that, in the above formula, $a$ is a non-limit ordinal, so $G_{a}$ is a onepoint-set.

It is tedious but not difficult to realize that $Y$ is a Tychonoff space and $X$ is closed in $Y$.

We claim that $F$ and $G$ cannot be separated in $Y$ by open sets. Take an arbitrary open set $U$ of $Y$ containing $F$. We shall prove that $\mathrm{Cl}_{Y} U \cap G \neq \emptyset$. Take for every limit $\alpha<\tau$ a point $x_{\alpha} \in F_{\alpha}$ and basic neighborhood $W^{\prime}\left(x_{\alpha}\right) \subset U$. Observe that, by definition of $F_{\alpha}$, the set $B_{\alpha}=\left\{b<\alpha: b\right.$ is non-limit and $\left.F_{b} \subset W\right\}$ is unbounded in $\alpha$. For every $\alpha$ pick an arbitrary $f(\alpha) \in B_{\alpha}$. Since for each $\alpha f(\alpha)<\alpha$, we can apply the Pressing-Down Lemma [Ku, Theorem 2.3] to find a non-limit $b<\tau$ so that $b=f(\alpha)$ for unboundedly (in $\tau$ ) many $\alpha$. We claim that for every $c<\tau$ there are $d>c$ and $\alpha \in f^{-1}(b)$ so that $(b, d) \in W^{\prime}\left(x_{\alpha}\right)$. Indeed, there is $\alpha \in f^{-1}(b)$ with $\alpha>c$. Since $B_{\alpha}$ is unbounded in $\alpha$, there is $d \in B_{\alpha}$ with $d>c$. Now both $b, d \in B_{\alpha}$, hence $(b, d) \in W^{\prime}\left(x_{\alpha}\right)$ and our claim holds. Next, consider $G_{b}$ and recall that, since $b$ is a non-limit ordinal, $G_{b}$ is a one-point-set, $G_{b}=\{g\}$. From the last claim it immediately follows that $g \in \mathrm{Cl}_{Y} U$, therefore $\mathrm{Cl}_{Y} U \cap G \neq \emptyset$ and the proof of Lemma 2.1 is complete. 
Lemma 2.6. If $X$ is weakly $C$-embedded in every larger Tychonoff space $Y$, containing $X$ as a closed subspace, then for every two disjoint completely separated closed subsets $P$ and $Q$ of $X$ either both are Lindelöf or one of them is compact.

Proof. Let $f: X \rightarrow \mathbb{R}$ be a continuous function such that $f(P)=\{0\}$ and $f(Q)=$ $\{1\}$. Suppose that $X$ is a closed subspace of a space $Y$. By our assumption, there is a function $\tilde{f}: Y \rightarrow \mathbb{R}$, which is continuous at every point of $X$ and satisfies $\tilde{f} \mid X=f$. Consequently, $P$ and $Q$ are separated by open sets in $Y$ and hence, by Lemma 2.1, either both $P$ and $Q$ are Lindelöf or one of them is compact.

Proof of Theorem 1.3. Sufficiency - the only part we need to prove - follows from Lemma 2.6 and the result of Blair, Hager and Johnson cited in the introduction.

Proof of Theorem 1.4. $1 \Rightarrow 2$ was observed in [AT]. $5 \Rightarrow 1$ follows from the result of Blair, Hager and Johnson, cited in the introduction. $2 \Rightarrow 3 \Rightarrow 4$ is evident. $4 \Rightarrow 5$ follows from Lemma 2.1 and the fact that $X$ is normal, being relatively normal in itself.

Proof of Theorem 1.1. $1 \Rightarrow 2$ is easy. $2 \Rightarrow 1$ follows from Lemma 2.1 for $P=X_{1}$, $Q=X_{2}$ and $X \oplus X$ "as" $X$.

\section{ACKNOWLEDGMENT}

The authors are strongly indebted to the referee for the helpful suggestions.

When this paper was already submitted we were informed that almost the same results were obtained independently by M.Matveev and O.Pavlov.

\section{REFERENCES}

[Ar] A.V.Arhangel'skij, Relative topological properties and relative topological spaces, Topology and Appl. 70 (1996), 87-89. MR 97f:54030

[AG] A.V.Arhangel'skij and H.M.M.Genedi, Beginnings of the theory of relative topological properties, General Topology, Spaces and Mappings, MGU, Moscow, 1989, pp. 87-89.

[AT] A. V. Arhangel'skij and J.Tartir, A characterization of compactness by a relative separation property, Q and A in Topology 14 (1996), 49-52. MR 96m:54037

[BH] R.L.Blair and A.W.Hager, Extensions of Zero-sets and of Real-valued Functions, Math. Z. 136 (1974), 41-52. MR 52:6652

[Bl] R.L.Blair, On $\nu$-embedded sets in topological spaces, Proceedings of the Second Pittsburgh Symposium on General Topology (Pittsburgh 1972), Springer-Verlag, Berlin, 1974. MR 50:11136

[HJ] A.W.Hager and D.G.Johnson, A note on certain subalgebras of $C(X)$, Canadian J. Math. 20 (1968), 389-393. MR 36:5697

[En] R.Engelking, General Topology, PWN, Warszawa, 1977. MR 58:18316b

[GJ] L.Gillman and M.Jerison, Rings of continuous functions, Springer-Verlag, Berlin, 1976. MR 53:11352

[He] E.Hewitt, A note on extensions of continuous functions, An. Acad. Brasil. Ci. 21 (1949), 175-179. MR 11:194c

$[\mathrm{Ku}]$ K. Kunen, Combinatorics, Handbook of Mathematical Logic (J. Barwise, eds.), Elsevier S.P., North-Holland, Amsterdam, 1977.

[Sm] Yu.Smirnov, Mappings of systems of open sets (in Russian), Matem. Sbornik 31 (1952), 152-166. MR 14:303g

[St] R. M. Stephenson, Jr, Initially k-compact and related spaces, Handbook of Set-Theoretic Topology (K.Kunen and J. Vaughan, eds.), Elsevier S.P., North-Holland, Amsterdam, 1984, pp. 603-632. MR 86i:54024 
[Wa] S. Watson, The Construction of Topological Spaces: Planks and Resolutions, Recent Progress in General Topology (M. Husek and J. van Mill, eds.), Elsevier S.P., North-Holland, Amsterdam, 1992, pp. 673-757. CMP 93:15

Dipartimento di Matematica, Citta Universitaria, Viale A.Doria 6, 95125, Catania, ITALY

E-mail address: bella@dipmat.unict.it

Moscow Center for Continuous Mathematical Education, B.Vlas'evskij Per. 11, 121002, Moscow, Russia

E-mail address: ivan@mccme.ru 